\title{
Radiation and Health Hazard
}

\author{
Ramesh Tripathi \\ Department of Physics, PN Campus, Pokhara \\ Email:trchandra3@yahoo.com
}

There are few areas with high levels of background radiation around the world, and epidemiological studies have indicated that natural radiation in these areas is not harmful for the inhabitants.

Eric J Hall, Professor of Radiology, College of Physicians and Surgeons, Columbia University, New York, write in his book entitled 'Radiation and Life' - "it Life on earth has developed with an ever present background of radiation. It is not something new, invented by the wit of man: radiation has always been there." Our world is radioactive and has been since it was created.

Sunshine is one of the most familiar forms of radiation. It delivers light, heat and suntans. We control our exposure to it with sunglasses, shade, hats, clothes and sunscreen. There would be no life on earth without lots of sunlight, but we have increasingly recognized that too much of it on our persons is not a good thing. Sunshine consists of radiation in a range of wavelengths from long-wave infra-red to shortwavelength ultraviolet, which creates the hazard. Over 60 radio nuclides (radioactive elements) can be found in nature, and they can be placed in three general categories:

Primordial - from before the creation of the Earth Cosmogenic - formed as a result of cosmic ray interactions

Human produced - enhanced or formed due to human actions (Minor amounts compared to natural)

Radio nuclides are found naturally in air, water and soil. They are even found in us, being that we are products of our environment. Every day, we ingest and inhale radio nuclides in our air and food and the water. Natural radioactivity is common in the rocks and soil that makes up our planet, in water and oceans, and in our building materials and homes. There is nowhere on Earth that you can not find
Natural Radioactivity.

Radioactive elements are often called radioactive isotopes or radio nuclides or just nuclides. There are over 1,500 different radioactive nuclides. Often, radio nuclides are symbolized based on the element and on the atomic weight, as in the case of radioactive hydrogen or tritium with an atomic weight of 3 is shown as $\mathrm{H}-3$ or $3 \mathrm{H}$. As another example, Uranium with the atomic weight of 235 would be shortened to U-235.

From the beginning, life has evolved in the presence of natural background ionizing radiation. The principal types and sources of such radiation are: (1) cosmic rays, which impinge on the Earth from outer space; (2) terrestrial radiations, which are released by the disintegration of radioisotopes. The source of terrestrial radiation includes the food, water, soil and building materials, which contain selected radioisotopes. From the space, about 100,000 cosmicrays neutrons and 400,000 secondary cosmic-rays pass through the average person per year. From the air, about 30,000 atoms (mainly Radon, Polonium, Bismuth and Lead) disintegrate each hour in the lung and give off alpha or beta particles and some gamma rays. From diet, about $15,000,000$ potassium-40 atoms disintegrate inside each individual every hour; all give off high energy beta particles and some emit gamma rays. Similarly, approximately 7,000 natural uranium atom disintegrate inside a person each hour, releasing alpha particles. From soil and building materials, more than 200,000,000 gamma rays pass through the average individual each hour (Britannica, 1994).

\section{High Natural Background Area}

The gamma radiation can be found in the environment even in the absence of geological deposits of radioactive materials or $\mathrm{K}, \mathrm{U}$ and $\mathrm{Th}$. Naturally occurring background radiation is the main source of exposure for most people. Levels typically range 
from about 1.5 to 3.5 millisievert per year but can be more than $50 \mathrm{mSv} / \mathrm{yr}$. The highest known level of background radiation affecting a substantial population is in Kerala and Madras States in India where some 140,000 people receive doses which average over 35 millisievert per year from gamma radiation. Comparable levels occur in Brazil and Sudan, with average exposures up to about $40 \mathrm{mSv} /$ yr to many people. Several places are known in Iran, India and Europe where natural background radiation gives an annual dose of more than $50 \mathrm{mSv}$ and up to $260 \mathrm{mSv}$ (at Ramsar in Iran). Lifetime doses from natural radiation range up to several thousand millisievert. However, there is no evidence of increased cancers or other health problems arising from these high natural levels. Thus, according to the ICRP recommendation, the dose up to $50 \mathrm{mSv} /$ $\mathrm{yr}$ is not harmful to the people if it comes from the nature as a background. (United Nations, 2000) Background radiation levels are from a combination of terrestrial (from the K-40, Th-232, Ra-226, etc.) and cosmic radiation (photons, muons, etc.). The level is fairly constant over the world, being 8-15 $\mu \mathrm{rad} / \mathrm{hr}$.

Around the world though, there are some areas with sizable populations that have high background radiation levels. The highest are found primarily in Brazil, India and China. The higher radiation levels are due to high concentrations of radioactive minerals in soil. One such mineral, Monazite, is a highly insoluble rare earth mineral that occurs in beach sand together with the mineral ilmenite, which gives the sands a characteristic color. The principal radionuclides in monazite are from the Th-232 series, but there is also some uranium its progeny, Ra-226.

In Brazil, the monazite sand deposits are found along certain beaches. The external radiation levels on these black sands range up to $5 \mathrm{mrad} / \mathrm{hr}(50 \mu \mathrm{Gy} /$ $\mathrm{hr}$ ), which is almost 400 times normal background in the US. Some of the major streets of the surrounding cites have radiation levels as high as $0.13 \mathrm{mrad} /$ $\mathrm{hr}(1.3 \mu \mathrm{Gy} / \mathrm{hr})$, which is more than 10 times the normal background. Another high background area in Brazil is the result of large rare earth ore deposits that form a hill that rises about 250 meters above the surrounding area. An ore body near the top of the hill is very near the surface, and contains an estimated 30,000 tons of thorium and 100,000 tons of rare earth elements. The radiation levels near the top of the hill are 1 to $2 \mathrm{mrad} / \mathrm{hr}(0.01$ to $0.02 \mathrm{mGy} / \mathrm{hr})$ over an area of about $30,000 \mathrm{~m} 2$. The plants found there have absorbed so much Ra 228, that they can will produce a self "X-ray" if placed on a sheet of photographic paper (an autoradiographed).

On the Southwest coast of India, the monazite deposits are larger than those in Brazil. The dose from external radiation is, on average, similar to the doses reported in Brazil, 500-600 mrad/yr (5 $6 \mathrm{mGy} / \mathrm{yr})$, but individual doses up to $3260 \mathrm{mrad} /$ yr (32.6 mGy/yr) have been reported. An area in China, has dose rates that is about $300-400 \mathrm{mrad} /$ yr (3-4 mGy/yr). This is also from monazite that contains Thorium,Uranium and Radium.

\section{Ionizing Radiation}

Ionizing radiation occurs in two forms, rays and particles, at the high frequency end of the energy spectrum. Ionizing radiation produces electricallycharged particles called ions in the materials it strikes. This process is called ionization. In the large chemical molecules of which all living things are made the changes caused may be biologically important. There are several types of ionizing radiation: X-rays and gamma rays, like light, represent energy transmitted in a wave without the movement of material, just as heat and light from a fire or the sun travels through space. X-rays and gamma rays are virtually identical except that X-rays are generally produced artificially rather than coming from the atomic nucleus. But unlike light, these rays have great penetrating power and can pass through the human body. Mass in the form of concrete, lead or water are used to shield us from them. Alpha particles consist of two protons and two neutrons, in the form of atomic nuclei. They thus have a positive electrical charge and are emitted from naturally occurring heavy elements such as uranium and radium, as well as from some man-made elements. Because of their relatively 
large size, alpha particles collide readily with matter and lose their energy quickly. They therefore have little penetrating power and can be stopped by the first layer of skin or a sheet of paper However, if alpha sources are taken into the body, for example by breathing or swallowing radioactive dust, alpha particles can affect the body's cells. Because they give up their energy over a relatively short distance, alpha particles inside the body can inflict more severe biological damage than other types of radiation.

Beta particles are fast-moving electrons ejected from the nuclei of atoms. These particles are much smaller than alpha particles and can penetrate up to 1 to 2 centimeters of water or human flesh. Beta particles are emitted from many radioactive elements. They can be stopped by a sheet of aluminum a few millimeters thick. Cosmic radiation consists of very energetic particles, mostly protons which bombard the earth from outer space. It is more intense at higher altitudes than at sea level where the earth's atmosphere is most dense and gives the greatest protection.

Neutrons are particles which are also very penetrating. On Earth they mostly come from the splitting, or fissioning, of certain atoms inside a nuclear reactor. Water and concrete are the most commonly used shields against neutron radiation from the core of the nuclear reactor. It is important to understand that alpha, beta, gamma and X-radiation do not cause the body or any other material to become radioactive. However, most materials in their natural state (including body tissue) contain measurable amounts of radioactivity.

\section{Measuring Ionizing Radiation}

The human senses cannot detect radiation or discern whether a material is radioactive. However, a variety of instruments can detect and measure radiation reliably and accurately. The amount of ionizing radiation, or 'dose', received by a person is measured in terms of the energy absorbed in the body tissue, and is expressed in gray. One gray (Gy) is one joule deposited per kilogram of mass.
Equal exposure to different types of radiation expressed as gray does not however necessarily produce equal biological effects. One gray of alpha radiation, for example, will have a greater effect than one gray of beta radiation. When we talk about radiation effects, we therefore express the radiation as effective dose, in a unit called the sievert (Sv). Regardless of the type of radiation, one sievert $(\mathrm{Sv})$ of radiation produces the same biological effect. Smaller quantities are expressed in 'millisievert' (one thousandth) or 'microsievert' (one millionth) of a sievert. We will use the most common unit, millisievert ( $\mathrm{mSv})$, here.

\section{Health Risks from Ionizing Radiation}

It has been known for many years that large doses of ionizing radiation, very much larger than background levels, can cause a measurable increase in cancers and leukemias ('cancer of the blood') after some years delay. It must also be assumed, because of experiments on plants and animals, that ionizing radiation can also cause genetic mutations that affect future generations, although there has been no evidence of radiation-induced mutation in humans. At very high levels, radiation can cause sickness and death within weeks of exposure. The degree of damage caused by radiation depends on many factors: dose, dose rate, type of radiation, the part of the body exposed, age and health, for example. Embryos including the human fetus are particularly sensitive to radiation damage. But what are the chances of developing cancer from low doses of radiation? The prevailing assumption is that any dose of radiation, no matter how small, involves a possibility of risk to human health. However there is no scientific evidence of risk at doses below about 50 millisieverts in a short time or about 100 millisieverts per year. At lower doses and dose rates, up to at least 10 millisieverts per year, the evidence suggests that beneficial effects are as likely as adverse ones. We are made up of chemicals, and it should be of no surprise that some of them are radio nuclides, many of which we ingest daily in our water and food. Table 2.1 shows the estimated concentrations of radio nuclides calculated for a $70 \mathrm{~kg}$ adult based ICRP guideline (United Nations, 
2000). Higher accumulated doses of radiation might produce a cancer which would only be observed several - up to twenty - years after the radiation exposure. This delay makes it impossible to say with any certainty which of many possible agents were the cause of a particular cancer. In western countries, about a quarter of people die from cancers, with smoking, dietary factors, genetic factors and strong sunlight being among the main causes. Radiation is a weak carcinogen, but undue exposure could certainly increase health risks. The body has defense mechanisms against damage induced by radiation as well as by chemical and other carcinogens. These can be stimulated by low levels of exposure, or overwhelmed by very high levels. On the other hand, large doses of radiation directed specifically at a tumour are used in radiation therapy to kill cancerous cells, and thereby often save lives (usually in conjunction with chemotherapy or surgery). Much larger doses are used to kill harmful bacteria in food, and to sterilise bandages and other medical equipment. Radiation has become a valuable tool in our modern world. See also The Peaceful Atom in this series. Tens of thousands of people in each technically advanced country work in medical and industrial environments where they may be exposed to radiation above background levels. Accordingly they wear monitoring 'badges' while at work, and their exposure is carefully monitored. The health records of these occupationally exposed groups often show that they have lower rates of mortality from cancer and other causes than the general public and, in some cases, significantly lower rates than other workers who do similar work without being exposed to radiation.

\section{ICRP Recommendation}

The following explanation deals the ICRP recommendation (United Nations, 2000) regarding the likely effects of a range of whole body radiation doses and dose rates to individuals: $10,000 \mathrm{mSv}$ (10 sieverts) as a short-term and whole-body dose would cause immediate illness, such as nausea and decreased white blood cell count, and subsequent death within a few weeks. Between 2 and 10 sieverts in a short-term dose would cause severe radiation sickness with increasing likelihood that this would be fatal. $1000 \mathrm{mSv}$ ( 1 sievert) in a short term dose is about the threshold for causing immediate radiation sickness in a person of average physical attributes, but would be unlikely to cause death. Above 1000 $\mathrm{mSv}$, severity of illness increases with dose. If doses greater than $1000 \mathrm{mSv}$ occur over a long period they are less likely to have early health effects but they create a definite risk that cancer will develop many years later. Above about $100 \mathrm{mSv}$, the probability of cancer (rather than the severity of illness) increases with dose. The estimated risk of fatal cancer is 5 of every 100 persons exposed to a dose of $1000 \mathrm{mSv}$ (ie. if the normal incidence of fatal cancer were $25 \%$, this dose would increase it to $30 \%$ ).

$50 \mathrm{mSv}$ is, conservatively, the lowest dose at which there is any evidence of cancer being caused in adults. It is also the highest dose which is allowed by regulation in any one year of occupational exposure. Dose rates greater than $50 \mathrm{mSv} / \mathrm{yr}$ arise from natural background levels in several parts of the world but do not cause any discernible harm to local populations. $20 \mathrm{mSv} / \mathrm{yr}$ averaged over 5 years is the limit for radiological personnel such as employees in the nuclear industry, uranium or mineral sands miners and hospital workers (who are all closely monitored). $10 \mathrm{mSv} / \mathrm{yr}$ is the maximum actual dose rate received by any Australian uranium miner. $3-5 \mathrm{mSv} / \mathrm{yr}$ is the typical dose rate (above background) received by uranium miners in Australia and Canada. $3 \mathrm{mSv} / \mathrm{yr}$ (approx) is the typical background radiation from natural sources in North America, including an average of almost $2 \mathrm{mSv} / \mathrm{yr}$ from radon in air. $2 \mathrm{mSv} /$ $\mathrm{yr}$ (approx) is the typical background radiation from natural sources, including an average of $0.7 \mathrm{mSv} / \mathrm{yr}$ from radon in air. This is close to the minimum dose received by all humans anywhere on Earth. 0.3-0.6 $\mathrm{mSv} / \mathrm{yr}$ is a typical range of dose rates from artificial sources of radiation, mostly medical. $0.05 \mathrm{mSv} / \mathrm{yr}$, a very small fraction of natural background radiation, is the design target for maximum radiation at the perimeter fence of a nuclear electricity generating station. In practice the actual dose is less.

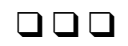

